\title{
Design of Permanent Magnets to Avoid Chaos in PM Synchronous Machines
}

\author{
Y. Gao, Student Member, IEEE, and K. T. Chau, Member, IEEE
}

\begin{abstract}
This paper analyzes the effect of permanent magnets (PMs) on the occurrence of chaos in PM synchronous machines (PMSMs). Based on the newly derived nonlinear system equation, the bifurcation analysis shows that the sizing of PMs significantly determines the stability of PMSMs. Hopf bifurcation and chaos may even occur in the PMSMs if the PMs are not properly sized. Experimental results of two practical PMSMs are provided to support the theoretical analysis.
\end{abstract}

Index Terms-Bifurcation, chaos, permanent magnet synchronous machine (PMSM).

\section{INTRODUCTION}

$\mathbf{T}$ I HE PERMANENT magnet synchronous machine (PMSM) is becoming more and more attractive for both industrial and electric vehicle applications [1] because of its inherent advantages of high-power density and high efficiency. However, investigation of its chaotic behavior has been surprisingly limited. Also, past discussion was based on ad hoc computer simulation, whereas the actual effect of PMs on the occurrence of chaos in PMSMs was unexplored [2].

This paper analyzes the relationship between sizing of PMs and chaos in PMSMs. Two practical PMSMs with different machine parameters are used for exemplification. Bifurcation analysis is employed to investigate the effect of PM sizing on chaotic behavior in PMSMs. Experimental verification of the theoretical results is described.

\section{SYSTEM MODELING}

Fig. 1 shows a PMSM driven by an induction motor (IM). The PMSM modeled in $d-q$ frame is given by [3]

$$
\left\{\begin{aligned}
L_{d s} \frac{d i_{d s}}{d t} & =-u_{d s}-R_{s} i_{d s}+n_{p} \omega_{r} L_{q s} i_{q s} \\
L_{q s} \frac{d i_{q s}}{d t} & =-u_{q s}-R_{s} i_{q s}-n_{p} \omega_{r} L_{d s} i_{d s}+n_{p} \psi_{a} \omega_{r} \\
J \frac{d \omega_{r}}{d t}= & T_{m}-\frac{3}{2} n_{p} \psi_{a} i_{q s}+\frac{3}{2} n_{p}\left(L_{d s}-L_{q s}\right) i_{d s} i_{q s} \\
& -B_{m} \omega_{r}
\end{aligned}\right.
$$

Manuscript received January 8, 2003. This work was supported by the Research Grants Council of Hong Kong Special Administrative Region, China.

The authors are with the Department of Electrical and Electronic Engineering, The University of Hong Kong, Hong Kong (e-mail: ktchau@eee.hku.hk; ygao@eee.hku.hk).

Digital Object Identifier 10.1109/TMAG.2003.816718

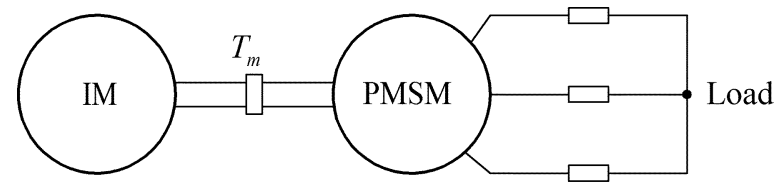

Fig. 1. PMSM system.

where $i_{d s}, i_{q s}$ are the stator currents, $u_{d s}, u_{q s}$ are the stator voltages, $L_{d s}$ and $L_{q s}$ are the stator inductances, $R_{s}$ is the stator resistance, $\psi_{a}$ is the PM flux, $n_{p}$ is the number of pole pairs, $\omega_{r}$ is the mechanical rotor speed, $T_{m}$ is the mechanical driving torque, $J$ is the rotor inertia, and $B_{m}$ the viscosity friction coefficient.

For simplicity, a symmetric load of resistance $R$ is adopted so that $u_{d s}$ and $u_{q s}$ can be expressed in terms of $R i_{d s}$ and $R i_{q s}$, respectively. In addition, the net driving torque is considered to be proportional to $i_{q s}$, namely, $T_{m}-T_{p m}=\mu i_{q s}$, where $T_{p m}=(3 / 2) n_{p} \psi_{a} i_{q s}$ is the PM torque and $\mu$ is a constant.

Equation (1) can be further simplified by transforming $t$ to $\tau t^{\prime}$, and $i_{d s}$ to $b k x, i_{q s}$ to $k y$, and $\omega_{r}$ to $z / \tau n_{p}$, where $b=$ $L_{q s} / L_{d s}, \tau=L_{q s} /\left(R_{s}+R\right)$, and $k$ is a positive constant. Hence, (1) can be expressed as

$$
\left\{\begin{array}{l}
\frac{d x}{d t^{\prime}}=-b x+y z \\
\frac{d y}{d t^{\prime}}=-y-x z+c z \\
\frac{d z}{d t^{\prime}}=a(\gamma k y-z)+\eta k^{2} x y
\end{array}\right.
$$

where $a=B_{m} \tau / J, c=\psi_{a} / k L_{q s}, \eta=3 n_{p}^{2}\left(L_{d s}-\right.$ $\left.L_{q s}\right) b \tau^{2} / 2 J$, and $\gamma=n_{p} \tau \mu / B_{m}$.

\section{PM SIZING}

According to [4], Hopf bifurcation and, hence, chaos exist in a dynamical system, such as the system described by (2), in three-dimensional state-space if its three eigenvalues are all nonzero with two of them being purely imaginary conjugates.

There are two major types of PMSMs: one with PMs mounted on the rotor surface, called the surface-magnet type; and one with PMs buried inside the rotor, called the interior-magnet type.

\section{A. Surface-Magnet PMSM}

The surface-magnet PMSM exhibits no saliency since $L_{d s}=$ $L_{q s}$. In order to avoid the trivial case when $\gamma=0, \gamma$ is set to be 
nonzero and $k$ is defined as $k=1 / \gamma$. Thus, (2) can be rewritten as

$$
\left\{\begin{array}{l}
\frac{d x}{d t^{\prime}}=-b x+y z \\
\frac{d y}{d t^{\prime}}=-y-x z+c z \\
\frac{d z}{d t^{\prime}}=a(y-z)
\end{array}\right.
$$

The fixed points can readily be solved by setting zero to the derivatives in (3). Although the origin is always a fixed point, it will never cause Hopf bifurcation since the characteristic equation of (3) evaluated at the origin is given by $(\lambda+b)\left[\lambda^{2}+(1+\right.$ a) $\lambda+a(1-c)]=0$ which will never have pure imaginary solution based on practical machine parameters. On the other hand, the characteristic equation evaluated at the nonzero fixed points $(c-1, \pm \sqrt{b(c-1)}, \pm \sqrt{b(c-1)})$ is

$$
\lambda^{3}+(a+b+1) \lambda^{2}+b(a+c) \lambda+2 a b(c-1)=0 .
$$

By applying the Routh-Hurwitz stability criterion, the critical value of $c$ is $c_{0}=a(a+b+3) /(a-b-1)$. Hence, the critical value of $\psi_{a}$ can be obtained as

$$
\psi_{a 0}=\frac{B_{m} L_{q s}^{2}\left[B_{m} L_{q s}+4 J\left(R+R_{s}\right)\right]}{\gamma J\left(R+R_{s}\right)\left[B_{m} L_{q s}-2 J\left(R+R_{s}\right)\right]}
$$

which denotes the maximum permissible value of $\psi_{a}$, hence the size of PMs, to avoid the occurrence of chaos. The corresponding eigenvalues are given by

$$
\lambda_{1}=-(a+b+1), \lambda_{2,3}= \pm j \sqrt{2 a b(a+1) /(a-b-1)} .
$$

Therefore, Hopf bifurcation occurs at $\psi_{a 0}$. Once $\psi_{a}$ exceeds this critical value, chaos may occur.

\section{B. Interior-Magnet PMSM}

The interior-magnet PMSM exhibits significant saliency since $L_{d s} \neq L_{q s}$, hence, offering additional salient power. In order to derive the explicit solution for the sizing of PMs, $\gamma$ is set to zero and $k$ is defined as $k=\sqrt{1 / \eta}$. Notice that $\gamma=0$ is the case when $T_{m}=T_{p m}$. Thus, (2) can be rewritten as

$$
\left\{\begin{array}{l}
\frac{d x}{d t^{\prime}}=-b x+y z \\
\frac{d y}{d t^{\prime}}=-y-x z+c z \\
\frac{d z}{d t^{\prime}}=x y-a z
\end{array}\right.
$$

By setting zero to all derivatives in (7), the fixed points can be easily derived, namely, the origin and the nonzero fixed-point pairs $\left(c / 2+\sqrt{\Delta} / 2, \pm \sqrt{a b}, x_{e q} y_{e q} / a\right)$ and $(c / 2-\sqrt{\Delta} / 2, \pm \sqrt{a b}$, $\left.x_{e q} y_{e q} / a\right)$, where $\Delta=c^{2}-4 a \geq 0$. The origin is always a stable fixed point since the corresponding eigenvalues are $-a$, $-b$, and -1 . The stability of those nonzero fixed points can be determined from the following characteristic equation:

$$
\lambda^{3}+(a+b+1) \lambda^{2}+b\left(a+x_{e q}^{2}\right) \lambda / a+2 b\left(x_{e q}^{2}-a\right)=0 .
$$

TABLE I

MACHINE PARAMETERS

\begin{tabular}{lll}
\hline \hline Parameter & Machine A & Machine B \\
\hline$L_{d s}$ & $11.5 \mathrm{mH}$ & $0.250 \mathrm{H}$ \\
$L_{q s}$ & $11.5 \mathrm{mH}$ & $0.210 \mathrm{H}$ \\
$R_{s}$ & $0.150 \Omega$ & $0.254 \Omega$ \\
$n_{p}$ & 4 & 3 \\
$\psi_{a}$ & $0.0283 \mathrm{~Wb}$ & $0.1336 \mathrm{~Wb}$ \\
$J$ & $1.440 \times 10^{-5} \mathrm{~kg} \cdot \mathrm{m}^{2}$ & $6.600 \times 10^{-4} \mathrm{~kg} \cdot \mathrm{m}^{2}$ \\
$B_{m}$ & $5.416 \times 10^{-4} \mathrm{~N} \cdot \mathrm{m} \cdot \mathrm{s}$ & $5.920 \times 10^{-3} \mathrm{~N} \cdot \mathrm{m} \cdot \mathrm{s}$ \\
\hline \hline
\end{tabular}

Hence, the Routh-Hurwitz stability condition is given by

$$
\left\{\begin{array}{l}
x_{e q}^{2}-a>0 \\
a(3 a+b+1)-x_{e q}^{2}(a-b-1)>0 .
\end{array}\right.
$$

Thus, it can be found that the fixed-point pair $(c / 2-\sqrt{\Delta} / 2$, $\left.\pm \sqrt{a b}, x_{e q} y_{e q} / a\right)$, if it exists, is always unstable and will never cause Hopf bifurcation since it fails to meet condition (9). However, it can be found that another fixed-point pair $(c / 2+\sqrt{\Delta} / 2$, $\left.\pm \sqrt{a b}, x_{e q} y_{e q} / a\right)$ can satisfy (9) with the critical value of $c$ equal to $c_{0}=4 a \sqrt{a /[(a-b-1)(3 a+b+1)]}$. Hence, the critical value of $\psi_{a}$ can be obtained as

$$
\psi_{a 0}=\frac{4}{n_{p}} \sqrt{\frac{2 B_{m}^{3} L_{d s} L_{q s} \tau}{3\left(L_{d s}-L_{q s}\right)\left(B_{m} \tau-J b-J\right)\left(3 B_{m} \tau+J b+J\right)}}
$$

The corresponding eigenvalues are given by

$\lambda_{1}=-(a+b+1), \lambda_{2,3}= \pm j \sqrt{b c_{0}\left(c_{0}+\sqrt{c_{0}^{2}-4 a}\right) / 2 a}$.

Therefore, Hopf bifurcation occurs at $\psi_{a 0}$. Once $\psi_{a}$ exceeds this critical value, chaos may occur.

\section{CHAOS}

As discussed, the sizing of PMs, namely of $\psi_{a}$, exhibits an important effect on the system behavior. Normal stable fixedpoint operation is guaranteed only when $\psi_{a}$ is lower than $\psi_{a 0}$. To assess the possible behavior beyond the stable region as defined by $\psi_{a 0}$, the bifurcation diagram is an important tool.

Two practical PMSMs (Machine A is a surface-magnet one, whereas Machine B is an interior-magnet one) with their parameters listed in Table I are used for exemplification. In order to experimentally verify the theoretical results based on these two PMSMs, $\gamma=10$ is chosen for Machine A, whereas $\gamma=0$ is for Machine B. Also, no load is adopted for both machines. Consequently, the corresponding bifurcation diagrams of $\omega_{r}$ via $\psi_{a}$ are determined as shown in Fig. 2. Although these two bifurcation diagrams have different patterns, a common conclusion can be drawn. Namely, both PMSMs will exhibit chaotic behavior once $\psi_{a}>\psi_{a 0}$. Whether the PMSM will operate at a fixed point or be trapped into chaos is mainly determined by the sizing of PMs. From Fig. 2 and Table I, it can be found that both Machines A and B will exhibit chaotic behavior since their PM flux values are both higher than their critical values (for Machine A: 


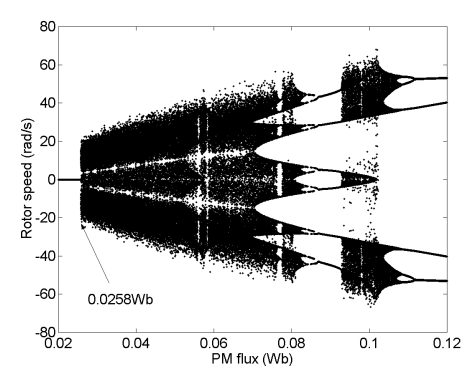

(a)

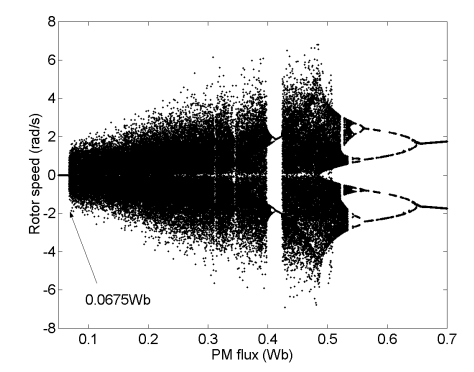

(b)

Fig. 2. Speed bifurcation diagrams. (a) Machine A. (b) Machine B.

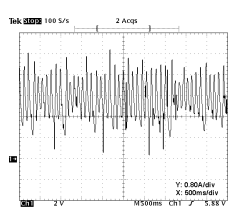

(a)

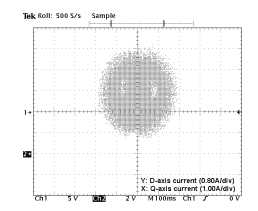

(d)

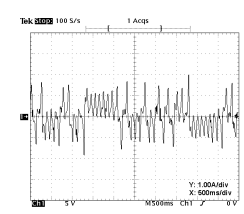

(b)

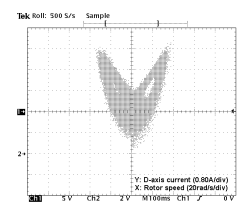

(e)

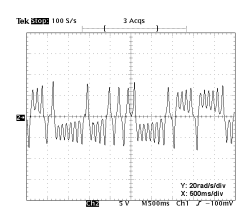

(c)

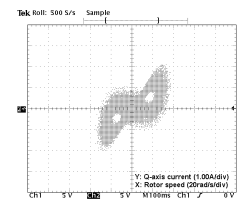

(f)
Fig. 3. Measured chaotic waveforms and trajectories of Machine A. (a) $i_{d s}$. (b) $i_{q s}$. (c) $\omega_{r}$. (d) $i_{d s}-i_{q s}$ plane. (e) $i_{d s}-\omega_{r}$ plane. (f) $i_{q s}-\omega_{r}$ plane.

$\psi_{a}=0.0283$ and $\psi_{a 0}=0.0258$; for Machine B: $\psi_{a}=0.1336$ and $\left.\psi_{a 0}=0.0675\right)$.

Figs. 3 and 4 are the measured time-domain waveforms of $i_{d s}, i_{q s}$ and $\omega_{r}$ as well as the measured trajectories of $i_{d s}-i_{q s}$, $i_{d s}-\omega_{r}$, and $i_{q s}-\omega_{r}$ planes for Machines A and B, respectively. It can be seen that both machines offer the well-known chaotic nature, namely random-like and bounded. In addition, the corresponding trajectories resemble a butterfly, which is actually the well-known Lorenz attractors.

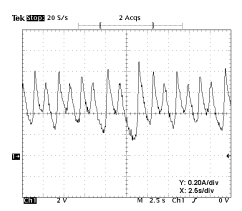

(a)

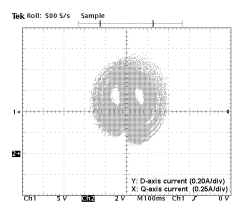

(d)

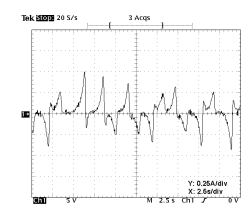

(b)

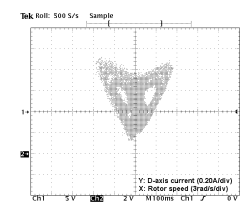

(e)

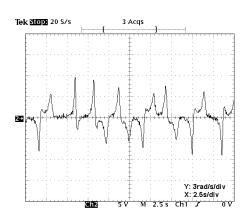

(c)

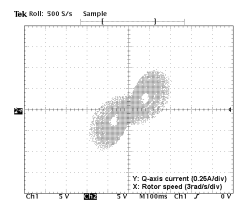

(f)
Fig. 4. Measured chaotic waveforms and trajectories of Machine B. (a) $i_{d s}$. (b) $i_{q s}$. (c) $\omega_{r}$. (d) $i_{d s}-i_{q s}$ plane. (e) $i_{d s}-\omega_{r}$ plane. (f) $i_{q s}-\omega_{r}$ plane.

Therefore, the sizing of PMs during the design of PMSMs should not be biased on the conventional optimization of power density, efficiency, or speed range but also need to avoid the occurrence of chaos based the newly derived equations of (5) and (10). Moreover, it should be noted that the proposed derivation and analysis can readily be extended to other general cases such as the presence of nonconstant load or even active load.

\section{CONCLUSION}

This paper has analyzed the effect of PMs on the occurrence of chaos in PMSMs, including both the surface-magnet and interior-magnet types. The key is derivation of the critical value of PM flux that can be offered by the PMSM. Beyond this critical value, Hopf bifurcation and, hence, chaos result. Two practical PMSMs have been employed for exemplification. The measured results, including both chaotic waveforms and trajectories, have verified the theoretical derivation and confirmed the importance of this analysis. Finally, the proposed analysis is so general that it can readily be extended to other modes of operation or even other motor drives.

\section{REFERENCES}

[1] J. Gan, K. T. Chau, C. C. Chan, and J. Z. Jiang, "A new surface-inset, permanent-magnet, brushless DC motor drive for electric vehicles," IEEE Trans. Magn., vol. 36, pp. 3810-3818, Sept. 2000.

[2] N. Hemanti, "Strange attractors in brushless dc motors," IEEE Trans. Circuits Syst. I, vol. 41, pp. 40-45, Jan. 1994.

[3] A. E. Fitzgerald, C. Kingsley Jr., and S. D. Umans, Electric Machinery. Singapore: McGraw-Hill, 1991.

[4] K. T. Alligood, T. D. Sauer, and J. A. Yorke, Chaos: An Introduction to Dynamical Systems. New York: Springer-Verlag, 1996. 